\title{
Understanding an emerging treatment population: Protocol for and baseline characteristics of a prospective cohort of people receiving treatment for pharmaceutical opioid dependence
}

Suzanne Nielsen ${ }^{1,2,13}$, Nicholas Lintzeris ${ }^{2}$, Bridin Murnion ${ }^{3}$, Louisa Degenhardt ${ }^{1}$, Raimondo Bruno $^{4}$, Paul Haber ${ }^{3}$, Jennifer Johnson ${ }^{5}$, Mark Hardy ${ }^{6}$, Stephen Ling ${ }^{7,8}$, Craig Saddler ${ }^{7,8,9}$, Adrian Dunlop ${ }^{9,10,12}$, Apo Demirkol ${ }^{2}$, Catherine Silsbury ${ }^{11}$, Nghi Phung ${ }^{11}$, Jennie Houseman ${ }^{6}$ and Briony Larance ${ }^{1}$

1. National Drug and Alcohol Research Centre, UNSW Sydney, Sydney, Australia;

2. South East Sydney Local Health District Drug and Alcohol Services, Sydney, Australia;

3. Discipline of Addiction Medicine, University of Sydney, Sydney, Australia;

4. School of Psychology, University of Tasmania, Hobart, Australia;

5. University Centre for Rural Health - North Coast, University of Sydney, Lismore, Australia;

6. Drug and Alcohol Services, Northern Sydney Local Health District, Sydney, Australia;

7. Drug and Alcohol Clinical Services, Hunter New England Local Health District, Newcastle, Australia;

8. University of Newcastle, Newcastle, Australia;

9. Cavalry Mater Hospital, Newcastle, Australia;

10. University of New South Wales, Sydney, Australia;

11. Drug Health Service, Western Sydney Local Health District, Sydney, Australia;

12. Priority Research Centre for Brain and Mental Health, University of Newcastle and Hunter Medical Research Institute, Newcastle, Australia.

13. Monash Addiction Research Centre, Monash University, Melbourne, Australia

Running title: Pharmaceutical Opioid Dependence Treatment Cohort Study

Corresponding author:

Dr Suzanne Nielsen

Monash Addiction Research Centre

Monash University, Peninsula Campus

PO Box 527, Frankston VIC 3199 Australia

Telephone no: +61 (3) 99044641

orcid.org/0000-0001-5341-1055

Email address: suzanne.nielsen@monash.edu

This is the author manuscript accepted for publication and has undergone full peer review but has not been through the copyediting, typesetting, pagination and proofreading process, which may lead to differences between this version and the Version of Record. Please cite this article as doi: 10.1111/dar.12859 


\begin{abstract}
Introduction and Aims: Despite large increases in pharmaceutical opioid dependence and related mortality, few studies have focussed on the characteristics and treatment experiences of those with pharmaceutical opioid dependence. We describe the formation of a prospective cohort of people receiving treatment for pharmaceutical opioid dependence and describe their baseline characteristics.
\end{abstract}

Design and Methods: People who had entered treatment pharmaceutical opioid dependence $(\mathrm{n}=108)$ were recruited through drug treatment services in New South Wales, Australia. We baseline characteristics of those that commenced pharmaceutical opioids for pain or other reasons and conducted a thematic analysis of responses to about treatment experience.

Results: Mean age was 41 years (SD 11), half were male (48\%). Just over half reported lifetime heroin use (57\%). Oxycodone (49\%) and codeine (29\%) were the most common opioids reported. Most (85\%) reported past-year problematic pain, 38\% reported chronic pain. Half (52\%) reported moderate to severe depression symptoms. Most (66\%) commenced opioids for pain, and this group were older, less likely to report a previous overdose and less likely to report use of illicit drugs compared to those commencing for other reasons. Five themes related to treatment expectations: (i) stigma; (ii) the restrictive nature of treatment; (iii) knowledge; (iv) pain; and (v) positive experience with buprenorphine.

Discussion and Conclusions: This study describes the complexities in an important emerging treatment population of pharmaceutical opioid dependent people. Findings highlights that addressing knowledge and perceptions around treatment may be critical to address the rising mortality associated with pharmaceutical opioid dependence.

Keywords: pharmaceutical opioid, codeine, oxycodone, buprenorphine, treatment 


\section{Background}

Within Australia and North America there have been steady increases in pharmaceutical opioid use and related harms [1-3]. These harms include large increases in opioid-related mortality [2, 4-6], and increasing treatment demand for dependence on pharmaceutical opioids $[1,7]$. More recently, higher potency opioids such as fentanyl have contributed to further sharp increases in mortality rates [8]. In the US, the prevalence of prescription opioid use disorder more than doubled from $1.4 \%$ to $2.9 \%$ in the 10 years leading up to 2013 , with fewer than one in five receiving treatment [9].

The understanding of opioid dependence among those prescribed opioids for chronic pain has changed dramatically over the past 10 years. Rates of dependence were initially thought to be low, estimated at 3\% of those exposed to chronic opioid therapy [10]. However, recent research has indicated that rates of dependence or 'addiction' are much higher than previously estimated [11]. An international systematic review suggested average rates of 'addiction' were 8 - 12\% of patients using long-term opioids for chronic pain (range, 95\% confidence interval 3-17\%) [12]. An Australian prospective cohort study demonstrated that $10-25 \%$ of chronic pain patients prescribed long-term opioids meet diagnostic criteria for dependence or addiction, depending on the definition used (different definitions e.g. ICD-10, DSM-IV, DSM-5 or 'Addiction' as defined by the American Pain Society capture different symptoms or numbers of symptoms, leading to these differences) [11].

Although the profile of people seeking treatment for pharmaceutical opioid dependence in Australia is not well described, one study identified a small, distinct group of pharmaceutical opioid dependent people who commonly initiated opioid use for pain, and had a lower prevalence of injection [13]. It is likely that people who are dependent on pharmaceutical opioids who seek treatment may have differing treatment profiles [13-15], and may also have differing treatment expectations, needs and preferences compared to people who are dependent on heroin, yet little work has examined this. Previous research highlights the importance of physical pain as a reason for opioid use among dependent people [16]. Understanding reasons for substance use is important to understand treatment needs, particularly for emerging populations such as those dependent on pharmaceutical opioids. 
In Australia, planned implementation of prescription drug monitoring [17, 18], combined with the removal of over-the-counter codeine in February 2018 [19] may result in increases in the number of people seeking treatment for pharmaceutical opioid dependence. Among those seeking treatment for pharmaceutical opioid dependence, it is expected that many will have started opioids in the context of pain management. As such, understanding this population and their treatment needs is a priority.

This paper describes the formation and baseline characteristics of a cohort of pharmaceutical opioid dependent people who entered treatment services for opioid dependence, with a view to:

1) Describe formation and characteristics of the cohort;

2) Explore how those that have commenced pharmaceutical opioids for pain treatment may differ from those that have commenced pharmaceutical opioid use for reasons other than pain; and

3) Understand the expectations and treatment experiences of those that enter treatment for opioid dependence where a pharmaceutical opioid is the main drug of concern.

\section{Methods}

Formation of the prospective cohort of people receiving treatment of pharmaceutical opioid dependence

\section{Cohort aims}

The overarching aims of the cohort study were to examine the characteristics and clinical trajectories of people receiving treatment for pharmaceutical opioid dependence over 2 years, including changes in opioid use, risk behaviour, physical health and mental health.

\section{Recruitment}

Participants were recruited from public and private drug and alcohol treatment services in six local health districts in metropolitan and regional New South Wales (NSW), Australia: 
Sydney, Western Sydney, Northern Sydney, South Eastern Sydney, Hunter New England and Northern NSW. These local health districts cover approximately half of the population of NSW. Ethical approval was received for all sites (X12-0372). Services included those providing opioid pharmacotherapy in primary care and public clinics, as well as those providing inpatient withdrawal management and outpatient withdrawal and counselling services.

\section{Eligibility}

Clinical staff at study sites identified potentially eligible participants. Those participants who gave consent for their contact details to be provided to the researchers were then contacted by a researcher who assessed their eligibility over the telephone, and gained informed consent where participants were eligible and willing to be involved in the study. The eligibility criteria were:

- Age e 18 years;

- Primary drug of concern relating to treatment was self-reported to be a pharmaceutical opioid;

- Sufficient English to provide informed consent to participate in study; and

- Able to provide contact details for follow up interviews.

One hundred and seventy-six people were referred to the study, with 26 not meeting eligibility criteria, 36 being unable to be contacted and 6 declining to participate, leaving 108 people interviewed at baseline.

\section{Interview procedures}

Structured interviews were conducted via telephone over four waves (at 0, 3, 12 and 24 months). Interviews (45 to 90 minutes' duration) were conducted by trained research assistants with medical or psychology backgrounds who had undergone suicide response training.

\section{Cohort retention and follow-up strategies}


There were a number of methods used to prevent attrition. A detailed locator form was completed at baseline with participants' details and two secondary contacts (e.g. family, medical professionals). Participants were reimbursed $\$ 40$ after each interview. Preferred contact methods were documented and flexible interview times were offered to maximise participation (e.g. evenings and weekends).

\section{Measures}

A complete description of the measures planned across the 4 waves of data collection are attached in Online Appendix A.

\section{Baseline description of cohort characteristics}

This study describes the clinical characteristics of the cohort using quantitative and qualitative data collected at the baseline assessment (Wave 0). Key measures to describe baseline characteristics include:

\section{Demographic characteristics}

Demographic information including age, gender, country of birth, relationship status, current employment status and highest education level attained was collected.

\section{Physical health}

Current self-reported health status was determined using the physical component scale from the SF-12 Health Survey with scores based on population norms with a mean score of 50 and a standard deviation of 10 [20]. Participants were asked whether they had experienced a number of other chronic health conditions as being problematic in the past 12 months (Yes/No), including stroke, heart attack, sleep apnoea, heart disease, high blood pressure, chronic lung disease, diabetes, epilepsy, cancer.

\section{Pain}

Three measures of pain were collected in this study: 
(1) Participants were asked whether they had experienced a number of problematic pain conditions in the past 12 months (Yes/No), including arthritis or rheumatism, chronic back or neck problems, frequent or severe headaches, visceral pain, generalised pain and other pain.

(2) Current pain (past 7 days) was assessed using the Brief Pain Inventory, which provides two scores on a continuous score out of 10 for current pain severity and pain interference $[21,22]$. A score of 1-4 is considered 'mild pain', 5-6 'moderate pain' and 7 or more 'severe pain' $[23,24]$.

(3) Participants were asked if they had a 'Current chronic pain' condition (Yes/No), defined as pain that had persisted for more than 3 months.

\section{Mental health}

Depression and generalised anxiety disorder were measured by the PHQ-9 and GAD-7 [25]. Previously validated cut-offs were used for screening tools as follows: symptoms indicating moderate to severe depression were defined as a score of e 10 on the PHQ-9 [26] and symptoms of moderate to severe anxiety were defined as a score of e 10 on the GAD-7 [27]. A cut-off score of e 3 on the Primary Care PTSD screener [28] was considered indicative of post-traumatic stress disorder. These scales are well-validated in a range of chronic pain and addiction populations [29-32].

\section{Substance use}

Lifetime and past month use of pharmaceutical opioids (non-medical), heroin, methamphetamine, cocaine, non-medical or non-prescribed benzodiazepines or other substances was captured. Where substance use was reported, participants were asked if the substance was ever injected. Where participants reported non-medical use of pharmaceutical opioids and heroin, age of initiation of each was collected, and order of use was calculated. Lifetime history of overdose was recorded.

Participants were asked 'What was the major reason you first used opioid analgesics' with several response options available, including: 'to relieve physical pain', 'to get high/for euphoria', 'to improve sleep', 'to relieve depression/sad feelings', 'to relieve nervousness, 
anxiety'; 'to deal with bad memories', 'to substitute for illicit opioids' or 'other'. These questions were drawn from questions previously used to explore reasons for initiation of prescribed opioids in other research with people dependent on prescription opioids $[16,33]$.

Participants were also asked about the main source of opioids when problematic use was emerging, including: 'doctor', 'pharmacy', 'given to them', 'stolen', 'prescribed where there was no legitimate medical reason', 'bought from a patient', 'family member' or 'dealer'.

\section{Prior and current substance use treatment experience}

Reasons for seeking treatment on this occasion, any previous treatment for opioid dependence, current treatment length and which pharmaceutical opioid was the main reason for entering treatment were documented.

\section{Treatment experience and expectations}

To enable an initial exploration of treatment experience and expectations, one open-ended question was asked: 'Do you have any comments about your expectation of treatment, or your experience receiving help for your problems with pharmaceutical opioids?' Participants' responses were documented by interviewers by typing summaries of what was said directly into the survey database, with key phrases documented verbatim.

\section{Data analyses}

Participants were assigned to two groups according to the major reason for their initial pharmaceutical opioid use; those who reported their first opioid analgesic use was 'To relieve physical pain' and those who reported 'Other reasons' (including 'to get high/for euphoria', 'to improve sleep', 'to relieve depression/sad feelings', 'to deal with bad memories', 'to substitute for illicit opioids', or 'other').

Participants reported the main source of opioids when problematic use was emerging and responses were then dichotomised according to whether they reported the source was a 'Medical source' (doctor or pharmacy for pain treatment), versus 'Other sources' (opioid analgesic was given to them, stolen, prescribed where there was no legitimate medical reason, 
bought from a patient, family member or dealer).

Odds ratios and 95\% confidence intervals were used for comparing the two groups of participants who reported initiating pharmaceutical opioid use 'to relieve physical pain' versus 'other reasons' on a range of demographic and clinical characteristics. Fisher's exact test was used where cell sizes of less than 5 were identified. Means, standard deviations and $\mathrm{t}$-tests were used where data were normal; medians, interquartile ranges and non-parametric statistics (independent samples Median Test) were used to compare groups where the distribution was non-normal.

We conducted a basic thematic analysis of responses to the open-ended question on treatment experience and expectations. The purpose was to provide an initial exploration to inform indepth investigation in future studies. The lead researcher (SN) initially read the responses and based on this conducted a first stage of the indicative analyses by developing a coding framework. Responses were then coded against and the coding framework and emerging themes were identified and developed [34]. The most common themes were reported, and where verbatim quotes were documented they were used to illustrate themes. Data were organised and coded with the assistance of an Excel spreadsheet.

\section{Results}

\section{Description of the cohort at baseline}

\section{Demographic characteristics}

The total sample had a mean age of 40.7 years, around half were male $(48 \%)$, one in three were employed or currently studying (30\%) and twelve identified as Aboriginal or Torres Strait Islander (11\%). Most (82\%) were born in Australia (Table 1). One in five had completed a university degree (19\%), and one in four had completed a TAFE/technical college certificate $(25 \%)$. One in three $(34 \%)$ was currently married or living in a de facto relationship. 


\section{Substance use history}

Just over half of the total sample reported ever injecting any drug (58\%). The most common substances participants reported having ever used were cannabis (86\%), meth/amphetamine $(72 \%)$ and cocaine $(62 \%)$. Past month use of meth/amphetamine and cocaine was reported by less than $10 \%$ of the cohort. Past month use of cannabis was reported by just under one third (30\%) and prescribed benzodiazepines (37\%) was reported by one in three participants. Just over half the sample reported a lifetime history of heroin use (57\%), with past month use reported by nine people.

\section{Pharmaceutical opioid initiation and source}

Two-thirds (66\%) of participants reported commencing pharmaceutical opioids 'to relieve physical pain'; the remainder reported commencing pharmaceutical opioids for 'other reasons' (44\%). At the time when self-identified problematic pharmaceutical opioid use began, the most common source of opioids was non-medical (i.e. not from a doctor, hospital or pharmacy for a therapeutic reason; Table 2).

For those that reported using both heroin and pharmaceutical opioids non-medically $(n=56)$, half $(\mathrm{n}=28,50 \%)$ reported that heroin use preceded pharmaceutical opioid use. A small group $(\mathrm{n}=8,14 \%)$ that reported commencing pharmaceutical opioids prior to heroin use, and a one in three commenced both in the same year $(n=20,36 \%)$.

\section{Physical health}

One in four participants (24\%) reported experiencing a physical health problem that was problematic in the past 12 months, most commonly sleep apnoea (16\%), high blood pressure $(11 \%)$, diabetes $(7 \%)$ and chronic lung disease $(6 \%)$.

\section{Pain}

Most of the cohort (85\%) reported a problematic pain condition in the past 12 months. The most common current pain conditions were chronic back or neck problems $(52 \%)$, followed by arthritis/rheumatism (27\%), frequent and severe headaches $(25 \%)$ and generalised pain $(25 \%)$. 
At cohort entry, $41 \%$ reported pain on the day of the interview. For those with current pain, the mean Brief Pain Inventory severity score of 6.0 (SD 2.3), and mean interference scores of 4.42 (SD 1.79) out of a possible 10. Forty-one participants (38\%) reported experiencing chronic pain at cohort entry.

\section{Mental health}

Half of the cohort (52\%) reported symptoms of current moderate to severe depression. Six in ten reported ever having suicidal thoughts $(60 \%)$. Four in ten $(39 \%)$ reported moderate to severe anxiety symptoms and one third met the cut-off for current PTSD on the Primary Care PTSD Screener (32\%).

\section{Treatment characteristics}

Forty-five participants (41\%) reported that their first treatment occasion was their current treatment episode, with median treatment duration of one year at cohort entry (Table 2). Although a range of main reasons for seeking treatment were reported, the most common was the participants own concern about their pharmaceutical opioid use (63\%). Oxycodone was the most frequent primary opioid of concern reported (49\%), with codeine (either prescribed or over the counter) next most often reported $(n=29 \%)$.

Just over half (55\%) of the participants were prescribed buprenorphine (either buprenorphine or buprenorphine-naloxone) and just over a third (37\%) were prescribed methadone at cohort entry. Just over one-third of the sample (37\%) were receiving counselling.

Four participants had completed treatment (e.g. inpatient detoxification) prior to the baseline interview and were not in any form of treatment at the first interview.

\section{Differences in cohort characteristics according to reasons for initiating pharmaceutical opioid use}

Compared to those who initiated pharmaceutical opioid use 'to relieve physical pain', the 'other reasons' group were significantly more likely to report: an extra-medical source for opioids when problematic use began (92\% vs $23 \%$ ), having ever used heroin ( $84 \%$ vs $45 \%$ ) and methamphetamine ( $84 \%$ vs $66 \%)$, having ever experienced an overdose ( $70 \%$ vs $45 \%)$, 
past month use of cannabis (43 vs. $23 \%$ ) and extra-medical opioid use in the past month (41\% vs $14 \%$; Table 1$)$.

The participants who reported initiating pharmaceutical opioid use 'To relieve physical pain' were significantly older, more likely to have current chronic pain, and reported poorer physical health (per the SF-12) than the 'Other reasons' group (Table 1). There were no differences in mental health characteristics, pharmacotherapy or counselling engagement between the two groups.

\section{Qualitative description of treatment experiences}

Participants were asked about their expectations of treatment or their experience of getting help with their pharmaceutical opioid problem. Seven participants did not provide a response to this question.

Five themes emerged from the data: (i) stigma; (ii) the restrictive nature of treatment; (iii) a lack of knowledge by patients and health care professionals; (iv) pain management; and (v) positive experience with buprenorphine products.

\section{Stigma}

Stigma was described in two different ways. Participants reported experiences of shame or stigma with respect to their experience in treatment. Participants expressed discomfort regarding hospital and clinic settings, for example one reported feeling 'bad' when he attended a clinic- 'I feel uncomfortable, because it's called a drug and alcohol place' (Male, 34 years). Some participants expressed preferences for pharmacy-based dosing for this reason. Concern was also expressed with being identified in drug treatment- 'I didn't want anyone to see me or know I was taking the Suboxone' (Female, 62 years). In addition to describing experiencing stigma, participants also expressed stigmatised views towards other treatment attendees. For example, one participant expressed frustration and wanted to be treated like 'a chronic pain patient and not a junkie' (Male, 33 years). 


\section{Restrictive nature of treatment}

Several participants commented on the restrictive nature of treatment including concerns regarding registration as an 'addict' (Female, 57 years), and referral to pharmacotherapy as a 'chemical prison' (Male, 34 years). Supervised pharmacotherapy treatment was noted to be 'inflexible' and 'expensive' by another (Female, 39 years). Nine participants said they planned to cease treatment soon, though two participants noted that, due to their pain, they were not confident that they would be able to cease treatment: 'If I can't bear the pain then I would like to stabilise on suboxone' (Female, 50 years).

\section{Lack of knowledge}

Participants described a personal lack of knowledge about treatment, and perceived health professionals also had a lack of knowledge about pharmaceutical opioid dependence and treatment options. This lack of knowledge was exemplified by one participant reporting a positive experience of treatment, despite reporting they initially 'didn't realise there was anything really helpful out there' (Female, 30 years) and another expressed frustration about a lack of knowledge 'I was as naive about opioid replacement treatment' (Female, 37 years). Another participant felt 'they [the doctors] don't know enough about the addiction to fix it' (Female, 32 years). One participant described that her doctor only knew where to refer her for treatment as the results of a coincidental conversation with another GP the previous day.

\section{Pain management}

Where patients commented on some aspect of pain $(n=12)$, six specifically noted their pain was reduced or adequately managed while taking methadone or buprenorphine 'the Suboxone has helped with my pain' (Male, 51 years). One participant had entered a pain management course through her treatment, which was viewed positively.

\section{Positive experiences}

A number of participants made more general comments that treatment was meeting, or exceeding, their expectations. Comments referring to specific buprenorphine product brand names described their experience with these products in positive terms and perceived them as life-saving: 'I was blown away, it was magic' (Female, 30). Buprenorphine-naloxone film was referred to as 'wonder-strips' (Male, 35 years). One participant highlighted how their 
treatment experience was more positive than anticipated: 'I expected it to be a bit scummier than it was, and the reality was that it was quite nice, and the doctor was very good.' (Male, 36 years).

\section{Discussion}

We describe a cohort of people in treatment for pharmaceutical opioid dependence. Most $(66 \%)$ commenced pharmaceutical opioid use in the context of treating pain, though chronic pain was described only by $38 \%$, suggesting that for some, pain was not the main reason for continuing to use opioids. More than half had symptoms of moderate to severe depression, previous suicidal thoughts and at least one previous overdose, highlighting that this is a treatment population with considerable complexity. These rates of co-morbidity are broadly comparable to those seen in other Australian substance use treatment populations $[35,36]$. Those with mental health co-morbidity may be at greater risk of dropping out of treatment [37], while treating depression may improve pain outcomes [38]. Taken together, this demonstrates the importance of assessing and responding to comorbidity. Open-ended responses demonstrated perceptions of treatment may act as barriers to treatment entry. These included stigma and lack of knowledge regarding treatment options. This was contrasted with largely positive experiences of treatment.

Half the sample were female, consistent with previous studies of pharmaceutical opioid dependence in Australia [15], but in contrast with heroin use where males are more likely to be represented [39]. This raises the question how services may respond to the unique needs of women (e.g. for women caring for children) and also how treatment responses may differ, given the known gender differences in the clinical profiles of opioid-dependent individuals [40].

Despite an average of one year in treatment at cohort entry, ongoing use of pharmaceutical opioids was reported by one in four. Benzodiazepine use was also reported by one in three. Benzodiazepine use in particular may contribute to overdose risk with heroin and 
pharmaceutical opioid use [41, 42]. This finding, combined with the common experience of overdose within the cohort, highlights that overdose prevention should be considered in all opioid dependent patients, irrespective of the type of opioid used or the reason for use. This is consistent with recognition of the importance of overdose prevention among chronic pain patients [43], with recent research confirming that offering naloxone to those using opioids for chronic pain would be well received [44].

The relatively high prevalence of lifetime heroin use and drug use by injection in this cohort is consistent with the findings of a previous study of pharmaceutical opioid dependence in Australia [13]. This demonstrates that many entering treatment have past experiences with illicit drug use, and raises the question as to whether those who do use illicit substances engage in existing drug treatment services. Research from the US indicates that a minority $(17 \%)$ of those with prescribed opioid use disorder have received treatment [9], though similar estimates from Australia do not exist.

Some differences were noted based on the reason for commencing pharmaceutical opioid use. At cohort entry, those commencing opioid use 'to relieve physical pain' were older, had greater current chronic pain and poorer physical health compared to those who commenced for 'other reasons'. Substance use histories also differed between these two groups, with less substance use by injection among those who initiated pharmaceutical opioid use 'to relieve physical pain'. Lifetime heroin and methamphetamine use in both groups were reported at rates higher than seen in those prescribed opioid for chronic pain [36], though recent heroin use overall was uncommon. A lower lifetime overdose history was noted among those commencing opioid 'to relieve physical pain' (compared to the 'other reasons' group); however, the proportion reporting a lifetime overdose was high in both groups, and of concern given that previous overdose is a known predictor of future overdose [45]. There were also many similarities indicating that although reasons for opioid exposure may differ, similarities exist in areas such as mental health and reasons for seeking treatment.

These findings also highlight the importance of structural factors that may contribute to the development of problematic pharmaceutical opioid (PO) use. Socioeconomic disadvantage 
and mental health comorbidity is associated with higher PO utilisation rates [46] and problematic opioid use and dependence [47]. There is substantial geographic variation in PO utilisation in Australia, with higher rates observed in areas outside of major cities [46, 48-50]. In part, this could be related to a lack of alternative pain treatment options in geographically remote areas. Australian studies indicate that people living with chronic non-cancer pain in rural and remote areas report a lack of access to pain specialists and financial barriers to pain treatment, compared with people from urban areas [51].

Open-ended questions revealed themes about treatment experience. Responses indicated that some treatment challenges previously described by people who are heroin dependent may equally affect those dependent on pharmaceutical opioids. These include stigma, cost and the inflexible nature of treatment $[52,53]$. Perceptions and expressions of stigma, and concern at being managed in identified 'drug treatment' services are consistent with those previously expressed by people dependent on over-the-counter codeine $[54,55]$. The perception that prescribers may not be knowledgeable about treatment of pharmaceutical opioid dependence was also consistent with previous research on over-the-counter codeine dependence [55], highlighting a need for targeted education for healthcare professionals regarding treatment of pharmaceutical opioid dependence. Despite these concerns, mostly positive experiences were described, suggesting that, treatments themselves may be acceptable largely to patients if barriers around treatment setting could be addressed. Pain was identified as an important aspect of treatment, though comments suggested pain was largely managed in the context of treatment for opioid dependence.

There are several caveats to consider when interpreting the data. The cohort was recruited primarily through drug and alcohol services, indicating that findings may not generalise to other settings. We did not establish a diagnosis of pharmaceutical opioid dependence using validated clinical interviews, and assumed that this diagnosis had been established prior to participants receiving treatment for pharmaceutical opioid dependence. As this is a treatment cohort, these population characteristics may not generalise to those that have not sought treatment. This highlights the importance of future research investigating the perspectives of those that have not entered treatment despite experiencing problems with pharmaceutical 
opioids. A related limitation is that, as the cohort were already in treatment, detailed changes in pain and mental health due to commencing treatment are not able to be examined. Third, the bivariate comparison of groups by reason for initiating opioid use reflects just one dimension of opioid use. Reasons for continuing to use pharmaceutical opioids, and the relationship between pharmaceutical opioid use and ongoing pain are also important facets of pharmaceutical opioid use. The reality is that there are many nuances to pharmaceutical opioid use and pain that are challenging to reflect in quantitative data. Fourth, there are limitations in the analyses of the single open-ended question, including that not all responses were recorded verbatim, and due to the nature of the survey in-depth discussion of responses was not possible [56]. As such, this data is not intended to represent a detailed qualitative exploration of these issues but represents a starting point for further exploration. Finally, the size of the cohort limited the statistical power.

\section{Conclusion}

In this sample of people in treatment for pharmaceutical opioid dependence, we found complex clinical presentations, and differences between groups based on the reasons pharmaceutical opioids were commenced. Participant comments indicted that addressing barriers to treatment, which include patient and health care provider knowledge and perceptions, may be critical if treatment is to address the rising mortality associated with pharmaceutical opioid dependence.

Acknowledgements: We wish to acknowledge the following people for their assistance with the study: David Helliwell, Amanda Brown, Rohan Holland, Simon Holliday, Glenys Dore, Carolyn Smith, Stefanie Leung, Bridin Murnion, Paul Haber, Trish Collie, Andrew Hallinan, and staff at the Kobi Clinic, Garden Court Clinic and Scott Street Clinic. Antonia Hordern, Michael Vanderhaven, Gina Lattas and Lexi Myers assisted with data collection. Kari Lancaster provided methodological advice on analyses of open-ended questions and comments on the manuscript. 
Funding sources: This study received funding from the Mental Health Drug and Alcohol Office of New South Wales. SN, BL and LD are supported by National Health and Medical Research Council research fellowships (\#1132433,\#1119992 and \#1041472). The National Drug and Alcohol Research Centre at the University of NSW is supported by funding from the Australian Government under the Substance Misuse Prevention and Service Improvements Grant Fund. 


\section{Reference:}

1. Nielsen S, Roxburgh A, Bruno R, Lintzeris N, Jefferson A, Degenhardt L. Changes in non-opioid substitution treatment episodes for pharmaceutical opioids and heroin from 2002 to 2011. Drug Alcohol Depend 2015;149:212-9.

2. Fischer B, Jones W, Rehm J. High correlations between levels of consumption and mortality related to strong prescription opioid analgesics in British Columbia and Ontario, 2005-2009. Pharmacoepidemiol Drug Saf 2013;22:438-42.

3. Guy GP Jr, Zhang K, Bohm MK, Losby J, Lewis B, Young R, Murphy LB, Dowell D.. Vital signs: Changes in opioid prescribing in the United States, 2006-2015. MMWR Morb Mortal Wkly Rep 2017;66:697-704.

4. Roxburgh A, Hall W, Burns L, Pilgrim J, Saar E, Nielsen S, Degenhardt L. Trends and characteristics of accidental and intentional codeine overdose deaths in Australia. Med J Aust 2015;203:299.

5. Roxburgh AaB, L. Accidental drug-induced deaths due to opioids in Australia, 2011. Sydney: National Drug and Alcohol Research Centre; 2015.

6. Rudd RA, Aleshire N, Zibbell JE, Gladden RM. Increases in Drug and Opioid Overdose Deaths--United States, 2000-2014. MMWR Morb Mortal Wkly Rep 2016;64:137882.

7. Alderks C. Trends in the use of methadone, buprenorphine, and extended-release naltrexone at substance abuse treatment facilities: 2003 - 2015 (update). The CBHSQ Report. Rockville (MD): Substance Abuse and Mental Health Services Administration (US); 20132017.

8. O'Donnell JK, Gladden RM, Seth P. Trends in Deaths Involving Heroin and Synthetic Opioids Excluding Methadone, and Law Enforcement Drug Product Reports, by Census Region - United States, 2006-2015. MMWR Morb Mortal Wkly Rep 2017;66:897-903. 9. Saha TD, Kerridge BT, Goldstein RB, Chou SP, Zhang H, Jung J, et al. Nonmedical prescription opioid use and DSM-5 nonmedical prescription opioid use disorder in the United States. J Clin Psychiatry 2016;77:772-80.

10. Fishbain DA, Cole B, Lewis J, Rosomoff HL, Rosomoff RS. What percentage of chronic nonmalignant pain patients exposed to chronic opioid analgesic therapy develop abuse/addiction and/or aberrant drug-related behaviors? A structured evidence-based review. Pain Med 2008;9:444-59.

11. Campbell G, Bruno R, Lintzeris N, Cohen M, Nielsen S, Hall W, et al. Defining problematic pharmaceutical opioid use among people prescribed opioids for chronic noncancer pain: do different measures identify the same patients? Pain 2016;157:1489-9 12. Vowles KE, McEntee ML, Julnes PS, Frohe T, Ney JP, van der Goes DN. Rates of opioid misuse, abuse, and addiction in chronic pain: a systematic review and data synthesis. Pain 2015; 156:569-76.

13. Nielsen S, Bruno R, Lintzeris N, Fischer J, Carruthers S, Stoove M. Pharmaceutical opioid analgesic and heroin dependence: how do treatment-seeking clients differ in Australia? Drug Alcohol Rev 2011;30:291-9. 
14. Brands B, Blake J, Sproule B, Gourlay D, Busto U. Prescription opioid abuse in patients presenting for methadone maintenance treatment. Drug Alcohol Depend 2004;73:199-207.

15. Nielsen S, Murnion B, Dunlop A, Degenhardt L, Demirkol A, Muhleisen P, Lintzeris N. Comparing treatment-seeking codeine users and strong opioid users: Findings from a novel case series. Drug Alcohol Rev 2015;34:304-11.

16. Weiss RD, Potter JS, Griffin ML, McHugh RK, Haller D, Jacobs P, et al. Reasons for opioid use among patients with dependence on prescription opioids: The role of chronic pain. J Subst Abuse Treat 2014;47:140-5.

17. State Government of Victoria. Real-time prescription monitoring Vic Health 2017 [Available from: https://www2.health.vic.gov.au/public-health/drugs-and-poisons/real-timeprescription-monitoring.

18. National approach to prescription drug misuse [press release]. 28/07/17 2017.

19. Therapeutic Goods Administration. Final decision on re-scheduling of codeine:

frequently asked questions 2016 [24/11/17]. Available from: https://www.tga.gov.au/finaldecision-re-scheduling-codeine-frequently-asked-questions.

20. Ware JE, Kosinski M, Keller SD. A 12-item short-form health survey: construction of scales and preliminary tests of reliability and validity. Med Care.1996;34:220-33.

21. Cleeland CS, Ryan KM. Pain assessment: Global use of the Brief Pain Inventory. Ann Acad Med Singapore 1994;23:129-38.

22. Cleeland C. The Brief Pain Inventory (BPI). 1991.

23. Serlin RC, Mendoza TR, Nakamura Y, Edwards KR, Cleeland CS. When is cancer pain mild, moderate or severe? Grading pain severity by its interference with function. Pain 1995;61:277-84.

24. Kapstad H, Hanestad BR, Langeland N, Rustøen T, Stavem K. Cutpoints for mild, moderate and severe pain in patients with osteoarthritis of the hip or knee ready for joint replacement surgery. BMC Musculoskelet Disord 2008;9:55.

25. Kroenke K, Spitzer RL, Williams JBW, Lowe B. The Patient Health Questionnaire Somatic, Anxiety, and Depressive Symptom Scales: a systematic review. Gen Hosp Psychiatry 2010;32:345-59.

26. Kroenke K, Spitzer R, Williams JBW. The PHQ-9: Validity of a brief depression severity measure. J Gen Intern Med 2001;16:606-13.

27. Spitzer RL, Kroenke K, Williams JBW, Lowe B. A brief measure for assessing generalized anxiety disorder: the GAD-7. Arch Intern Med 2006;166:1092-7.

28. Prins A, Ouimette P, Kimerling R, Cameron RP, Hugelshofer DS, Shaw-Hegwer J, et al. The primary care PTSD screen (PC-PTSD): Development and operating characteristics Primary Care Psychiatry 2003;9:9-14.

29. Seo JG, Park SP. Validation of the Patient Health Questionnaire-9 (PHQ-9) and PHQ2 in patients with migraine. J Headache Pain 2015;16:65.

30. Kiely KM, Butterworth P. Validation of four measures of mental health against depression and generalized anxiety in a community based sample. Psychiatry Res

2015;225:291-8. 
31. Delgadillo J, Payne S, Gilbody S, Godfrey C, Gore S, Jessop D, Dale V. How reliable is depression screening in alcohol and drug users? A validation of brief and ultra-brief questionnaires. J Affect Disord 2011;134:266-71.

32. Choi Y, Mayer TG, Williams MJ, Gatchel RJ. What is the best screening test for depression in chronic spinal pain patients? Spine 2014;14:1175-82.

33. Weiss RD, Potter JS, Fiellin D, Byrne M, Connery HS, Dickinson W, et al. A TwoPhase Randomized Controlled Trial of Adjunctive Counseling during Brief and Extended Buprenorphine-Naloxone Treatment for Presciption Opioid Dependence. Arch Gen Psychiatry. 2011;68:1238-46.

34. Braun V, Clarke V. Using thematic analysis in psychology. Qual Res Psychol 2006;3:77-101.

35. Smith K, Mattick R, Bruno R, Nielsen S, Cohen M, Campbell G, et al. Factors associated with the development of depression in chronic non-cancer pain patients following the onset of opioid treatment for pain. J Affect Disord 2015;184:72-80.

36. Campbell G, Nielsen S, Bruno R, Lintzeris N, Cohen M, Hall W, et al. The Pain and Opioids IN Treatment (POINT) study: Characteristics of a cohort using opioids to manage chronic non-cancer pain. Pain 2015;156:231-42.

37. Krawczyk N, Feder KA, Saloner B, Crum RM, Kealhofer M, Mojtabai R. The association of psychiatric comorbidity with treatment completion among clients admitted to substance use treatment programs in a U.S. national sample. Drug Alcohol Depend 2017; 175:157-63.

38. Teh CF, Zaslavsky A, Reynolds CF, Cleary PD. Effect of depression treatment on chronic pain outcomes. Psychosom Med 2010;72:61-7.

39. Degenhardt L, Whiteford HA, Ferrari AJ, Baxter AJ, Charlson FJ, Hall WD, et al. Global burden of disease attributable to illicit drug use and dependence: findings from the Global Burden of Disease Study 2010. Lancet 2013;382:1564-74.

40. Back SE, Payne RL, Wahlquist AH, Carter RE, Stroud Z, Haynes L, et al. Comparative profiles of men and women with opioid dependence: results from a national multisite effectiveness trial. Am J Drug Alcohol Abuse 2011;37:313-23.

41. Jones CM, McAninch JK. Emergency Department Visits and Overdose Deaths From Combined Use of Opioids and Benzodiazepines. Am J Prev Med 2015;49:493-501.

42. Dietze P, Jolley D, Fry C, Bammer G. Transient changes in behaviour lead to heroin overdose: results from a case-crossover study of non-fatal overdose. Addiction 2005; 100:636-42.

43. Coe MA, Walsh SL. Distribution of naloxone for overdose prevention to chronic pain patients. Prev Med 2015;80:41-3.

44. Nielsen S, Peacock A, Lintzeris N, Bruno R, Larance B, Degenhardt L. Knowledge of opioid overdose and attitudes to supply of take-home naloxone among people with chronic noncancer pain prescribed opioids. Pain Med 2018;19:533-40.

45. Coffin PO, Tracy M, Bucciarelli A, Ompad D, Vlahov D, Galea S. Identifying injection drug users at risk of nonfatal overdose. Acad Emerg Med 2007;14:616-23.

46. Degenhardt L, Gisev N, Cama E, Nielsen S, Larance B, Bruno R. The extent and correlates of community-based pharmaceutical opioid utilisation in Australia.

Pharmacoepidemiol Drug Saf 2016;25:521-38. 
47. Campbell G, Nielsen S, Larance B, Bruno R, Mattick R, Hall W, et al. Pharmaceutical Opioid Use and Dependence among People Living with Chronic Pain: Associations Observed within the Pain and Opioids in Treatment (POINT) Cohort. Pain Med 2015;16:1745-58.

48. Gisev N, Nielsen S, Cama E, Larance B, Bruno R, Degenhardt L. An ecological study of the extent and factors associated with use of prescription and over-the-counter codeine in Australia. Eur J Clin Pharmacol 2016;72:469-94.

49. Berecki-Gisolf J, Hassani-Mahmooei B, Clapperton A, McClure R. Prescription opioid dispensing and prescription opioid poisoning: Population data from Victoria, Australia 2006 to 2013. Aust N Z J Public Health 2017;41:85-91.

50. Rintoul AC, Dobbin MDH, Drummer OH, Ozanne-Smith J. Increasing deaths involving oxycodone, Victoria, Australia, 2000-09. Inj Prev 2011;17:254-9.

51. Peacock A, Nielsen S, Bruno R, Campbell G, Larance B, Degenhardt L. Geographic variation in health service use and perceived access barriers for Australian adults with chronic non-cancer pain receiving opioid therapy. Pain Med 2016;17:2003-16.

52. Crawford S. Shouting through bullet-proof glass: Some reflections on pharmacotherapy provision in one Australian clinic. Int J Drug Policy 2013;24:e14-e7.

53. Fischer B, Chin AT, Kuo I, Kirst M, Vlahov D. Canadian illicit opiate users' views on methadone and other opiate prescription treatment: an exploratory qualitative study. Subst Use Misuse. 2002;37:495-522.

54. Van Hout MC, Horan A, Santlal K, Rich E, Bergin M. 'Codeine is my companion': misuse and dependence on codeine containing medicines in Ireland. Ir J Psychol Med 2015:114.

55. Cooper RJ. 'I can' t be an addict. I am.' Over-the-counter medicine abuse: A qualitative study. BMJ Open. 2013;:e002913. doi: 10.1136/bmjopen-2013-002913 56. O'Cathain A, Thomas KJ. "Any other comments?" Open questions on questionnaires - a bane or a bonus to research? BMC medical research methodology. 2004;4:25. 
Table 1: Demographic, health and substance use characteristics at cohort entry by reason for first pharmaceutical opioid use $(n=108)$

\begin{tabular}{|c|c|c|c|c|c|c|}
\hline & $\begin{array}{c}\text { A. Total } \\
\text { sample } \\
(\mathrm{N}=108) \\
\%(\mathrm{n})\end{array}$ & $\begin{array}{c}\text { B. To relieve } \\
\text { physical pain } \\
n=71 \\
\%(n)\end{array}$ & $\begin{array}{l}\text { C. Other } \\
\text { reason } \\
(n=37) \\
\%(n)\end{array}$ & $\begin{array}{l}\text { Odds } \\
\text { ratio/t }\end{array}$ & $\begin{array}{c}\text { B vs C } \\
95 \% \mathrm{CI}, \mathrm{df}\end{array}$ & $P$ \\
\hline Mean age (SD) & $40.7(10.54)$ & $42.6(10.0)$ & $36.9(10.6)$ & 1.06 & $1.02,1.10$ & 0.009 \\
\hline Male & $48.1(52)$ & $45.1(32)$ & $54.1(20)$ & 1.43 & $0.65,3.18$ & 0.375 \\
\hline Employed/student & $29.6(32)$ & $29.6(21)$ & $29.7(11)$ & 0.99 & $0.42,2.37$ & 0.987 \\
\hline Born in Australia & $81.5(88)$ & $83.1(59)$ & $78.4(29)$ & 1.36 & $0.50,3.68$ & 0.549 \\
\hline $\begin{array}{l}\text { Highest level of education } \\
\text { attained year } 10 \text { or less }\end{array}$ & $47.2(51)$ & $43.7(31)$ & $54.1(20)$ & 0.65 & $0.30,1.46$ & 0.304 \\
\hline Married/de facto relationship & $34.3(37)$ & $33.8(24)$ & $35.1(13)$ & 1.06 & $0.46,2.45$ & 0.890 \\
\hline Current chronic pain & $38.0(41)$ & $52.1(37)$ & $10.8(4)$ & 8.98 & $2.88,28.00$ & $<0.001$ \\
\hline $\begin{array}{l}\text { Physical health functioning (SF- } \\
12)^{\mathrm{a}} \text { Mean (SD) }\end{array}$ & $40.4(10.6)$ & $37.9(10.7)$ & $45.1(8.7)$ & 3.504 & & 0.001 \\
\hline \multicolumn{7}{|l|}{ Mental health } \\
\hline Moderate to severe depression $^{b}$ & $51.9(56)$ & $53.5(38)$ & $48.6(18)$ & 1.22 & $0.55,2.69$ & 0.631 \\
\hline Moderate to severe anxiety ${ }^{\mathrm{b}}$ & $38.9(42)$ & $38.0(27)$ & $40.5(15)$ & 0.90 & $0.40,2.03$ & 0.799 \\
\hline Post-traumatic stress disorder & $32.4(35)$ & $26.8(19)$ & $43.2(16)$ & 0.48 & $0.21,1.11$ & 0.082 \\
\hline Ever had suicidal thoughts & $59.8(64)$ & $58.6(41)$ & $62.2(23)$ & 0.86 & $0.38,1.95$ & 0.719 \\
\hline \multicolumn{7}{|l|}{ Substance use (illicit) } \\
\hline Ever injected any drug & $58.3(63)$ & $45.1(32)$ & $83.8(31)$ & 0.16 & $0.06,0.43$ & $<0.001$ \\
\hline \multicolumn{7}{|l|}{ Heroin use } \\
\hline Lifetime (\%) & $57.0(61)$ & $42.9(30)$ & $83.8(31)$ & 0.15 & $0.05,0.39$ & $<0.001$ \\
\hline Past month (\%) & $8.3(9)$ & $5.6(4)$ & $13.5(5)$ & 0.38 & $0.10,1.52$ & 0.269 \\
\hline \multicolumn{7}{|l|}{ Meth/amphetamine use } \\
\hline Lifetime (\%) & $72.0(77)$ & $65.7(46)$ & $83.8(31)$ & 0.37 & $0.14,1.01$ & 0.048 \\
\hline Past month (\%) & $9.3(10)$ & $5.6(4)$ & $16.2(6)$ & 0.31 & $0.08,1.17$ & 0.088 \\
\hline \multicolumn{7}{|l|}{ Cocaine use } \\
\hline Lifetime (\%) & $61.7(66)$ & $57.1(40)$ & $70.3(26)$ & 0.56 & $0.24,1.32$ & 0.184 \\
\hline Past month (\%) & $8.3(9)$ & $7.0(5)$ & $10.8(4)$ & 0.25 & $0.22,2.85$ & 0.270 \\
\hline \multicolumn{7}{|l|}{ Cannabis use } \\
\hline Lifetime (\%) & $86.0(92)$ & $82.9(58)$ & $91.9(34)$ & 0.43 & $0.11,1.62$ & 0.200 \\
\hline Past month (\%) & $29.6(32)$ & $22.5(16)$ & $43.2(16)$ & 0.38 & $0.16,0.90$ & 0.025 \\
\hline \multicolumn{7}{|l|}{ Nicotine use } \\
\hline Lifetime (\%) & $92.5(99)$ & $91.4(64)$ & $94.6(35)$ & 0.61 & $.12,3.18$ & 0.554 \\
\hline Past month (\%) & $72.0(77)$ & $86.6(48)$ & $78.4(29)$ & 0.60 & $.24,1.53$ & 0.554 \\
\hline \multicolumn{7}{|l|}{ Alcohol use } \\
\hline Lifetime (\%) & $97.2(104)$ & $95.7(67)$ & $100.0(37)$ & - & - & 0.202 \\
\hline Past month $(\%)$ & $43.0(46)$ & $37.1(26)$ & $54.1(20)$ & .502 & $0.22,1.13$ & 0.09 \\
\hline \multicolumn{7}{|l|}{ Substance use (pharmaceutical) } \\
\hline Past month prescribed BZD (\%) & $37.0(40)$ & $38.0(27)$ & $35.1(13)$ & 1.13 & $0.50,2.60$ & 0.768 \\
\hline
\end{tabular}




$\begin{array}{lcccccc}\begin{array}{l}\text { Past month extramedical PO } \\ (\%)\end{array} & 23.1(25) & 14.1(10) & 40.5(15) & 0.24 & 0.09,0.61 & 0.002 \\ \quad \text { Lifetime overdose history } & 53.7(58) & 45.1(32) & 70.3(26) & 0.35 & 0.15, .081 & 0.013 \\ \quad \text { Lifetime overdoses (Median, } & 1(2) & 0(2) & 2(3) & - & - & 0.063 \\ \text { IQR) }^{\mathrm{c}}\end{array}$

${ }^{a}$ Australian normative data are a mean of 50 with a standard deviation of $10 .{ }^{b} \mathrm{Cut}-0 \mathrm{ff}$ of 10 or more on the GAD/PHQ. ${ }^{\mathrm{c}}$ Independent samples Median Test. BZD, benzodiazepine; CI, confidence interval; df, degrees of freedom for $\mathrm{X}^{2}$ test; IQR, interquartile range; PO, pharmaceutical opioid. One participant did not answer substance use questions. 
Table 2: Opioid use and drug treatment history

\begin{tabular}{|c|c|c|c|c|c|c|}
\hline & & & & \multicolumn{3}{|c|}{ B vs. C } \\
\hline & $\begin{array}{l}\text { A. Whole } \\
\text { Sample } \\
(\mathrm{N}=108) \\
\%(\mathrm{n})\end{array}$ & $\begin{array}{l}\text { B. To relieve } \\
\text { physical } \\
\text { pain }(n=71) \\
\%(n)\end{array}$ & $\begin{array}{l}\text { C. Other } \\
\text { reason } \\
(\mathrm{n}=37) \\
\%(\mathrm{n})\end{array}$ & $\underset{2}{\mathrm{OR} / \mathrm{X}}$ & $95 \% \mathrm{CI} / \mathrm{df}$ & $P$ \\
\hline \multirow{2}{*}{\multicolumn{4}{|c|}{$\begin{array}{l}\text { Opioid source when problematic } \\
\text { use began }\end{array}$}} & 15.49 & $4.35,55.20$ & $<\mathbf{0 . 0 0}$ \\
\hline & & & & & & 1 \\
\hline Medical source for therapeutic & $40.7(44)$ & $57.7(41)$ & $8.1(3)$ & & & \\
\hline \multicolumn{7}{|l|}{ use } \\
\hline Other source ${ }^{\mathrm{a}}$ & $59.3(64)$ & $23.3(30)$ & $91.9(34)$ & & & \\
\hline Current treatment first episode & $41.7(45)$ & $45.1(32)$ & $35.1(13)$ & 1.52 & $0.67,3.44$ & 0.32 \\
\hline $\begin{array}{l}\text { Current treatment length [Median } \\
\text { months }(\mathrm{IQR})](\mathrm{n}=104)^{\wedge}\end{array}$ & $12.0(21.6)$ & $12.0(21.6)$ & $9.6(21.6)$ & $\wedge$ & $\wedge$ & 0.41 \\
\hline \multicolumn{7}{|l|}{ Main reason for seeking treatment } \\
\hline Concerns about own PO use & $63.0(68)$ & $57.7(41)$ & $73.0(27)$ & 0.50 & $0.21,1.20$ & 0.12 \\
\hline $\begin{array}{l}\text { Difficulty sourcing opioids (e.g. } \\
\text { prescriber refusal) }\end{array}$ & $12.0(13)$ & $15.5(11)$ & $5.4(2)$ & 3.21 & $.67,15.32$ & 0.21 \\
\hline $\begin{array}{l}\text { Other health or lifestyle } \\
\text { concerns }\end{array}$ & $25.0(27)$ & $26.8(19)$ & $21.6(8)$ & 1.33 & $.52,3.40$ & 0.56 \\
\hline \multicolumn{7}{|l|}{$\begin{array}{l}\text { Pharmaceutical opioid leading to } \\
\text { treatment }\end{array}$} \\
\hline Oxycodone & $49.1(53)$ & $47.9(34)$ & $54.1(19)$ & .87 & $.39,1.93$ & 0.73 \\
\hline Prescribed codeine & $7.4(8)$ & $11.3(8)$ & 0 & - & - & 0.05 \\
\hline Over the counter codeine & $21.3(23)$ & $21.1(15)$ & $21.6(8)$ & .97 & $.37,2.56$ & 0.95 \\
\hline Other (e.g. morphine, fentanyl) & $24(22.2)$ & $14(19.7)$ & $10(27.0)$ & .77 & $.12,4.84$ & 0.78 \\
\hline \multicolumn{7}{|l|}{ Opioid treatment at cohort entry } \\
\hline Methadone & $37.0(40)$ & $31.0(22)$ & $48.6(18)$ & 0.47 & $0.21,1.07$ & 0.07 \\
\hline Buprenorphine & $54.6(59)$ & $56.3(40)$ & $51.4(19)$ & 1.22 & $0.55,2.71$ & 0.62 \\
\hline Counselling at cohort entry ${ }^{\mathrm{c}}$ & $37.0(40)$ & $38.0(27)$ & $35.1(13)$ & 1.33 & $0.50,2.59$ & 0.87 \\
\hline
\end{tabular}




\section{University Library}

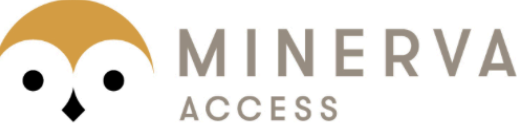

A gateway to Melbourne's research publications

Minerva Access is the Institutional Repository of The University of Melbourne

\section{Author/s:}

Nielsen, S;Lintzeris, N;Murnion, B;Degenhardt, L;Bruno, R;Haber, P;Johnson, J;Hardy, M;Ling, S;Saddler, C;Dunlop, A;Demirkol, A;Silsbury, C;Phung, N;Houseman, J;Larance, B

Title:

Understanding an emerging treatment population: Protocol for and baseline characteristics of a prospective cohort of people receiving treatment for pharmaceutical opioid dependence

\section{Date:}

2018-11-01

\section{Citation:}

Nielsen, S., Lintzeris, N., Murnion, B., Degenhardt, L., Bruno, R., Haber, P., Johnson, J., Hardy, M., Ling, S., Saddler, C., Dunlop, A., Demirkol, A., Silsbury, C., Phung, N., Houseman, J. \& Larance, B. (2018). Understanding an emerging treatment population: Protocol for and baseline characteristics of a prospective cohort of people receiving treatment for pharmaceutical opioid dependence. DRUG AND ALCOHOL REVIEW, 37 (7), pp.887-896. https://doi.org/10.1111/dar.12859.

Persistent Link:

http://hdl.handle.net/11343/284555 